\title{
LA VISIÓN DEL OTRO: LOS SALVAJES CIVILIZADOS DE WILLIAM GOLDING
}

\author{
Ángel Mateos-Aparicio Martín-Albo. Universidad de Castilla-La Mancha
}

\begin{abstract}
La representación de la alteridad es una de los problemáticas más señaladas por las teorías literarias más recientes. El postmodernismo y el postcolonialismo le han prestado atención especial desde un punto de vista cultural y literario. Destaca entre ellos el trabajo integrador de Homi Bhabha, quien incide en el análisis del espacio indefinido entre extremos. Con estos avances recientes, esperamos perfeccionar la interpretación de las primeras novelas de William Golding, en la aproximación a los puntos de partida ideológicos y el estudio de las técnicas literarias y estilísticas más innovadoras.
\end{abstract}

One of our [England's] faults is to believe that evil is somewhere else and inherent in another nation.

(Golding, The Hot Gates)

Cuando William Golding comenta las razones que le impulsaron a escribir la que sería su primera y más famosa novela, Lord of the Flies, afirma con cierta ironía:

We had just put the children to bed after reading to the elder some adventure story or other. (...) But I was tired of these islands with their paper-cutout goodies and baddies and eveything for the best in the best of all possible worlds. I said to my wife, "Wouldn't it be a good idea if I wrote a story about boys on an island and let them behave the way they really would?"

(A Moving Target, 163)

Definitivamente fue una excelente idea introducir los estereotipos en un laboratorio y proceder a una concienzuda disección. El resultado fue parecido al que se obtiene si se analiza empíricamente el truco visual de un prestidigitador: pronto aparecen los hilos escondidos que mantenían engañada nuestra percepción. Detrás del estereotipo surgió todo un entramado de hilos culturales que habían sido tejidos durante mucho tiempo por la 
ciencia y la cultura occidentales. Las representaciones estereotípicas se sostenían de una manera tal vez precaria pero efectiva ante unos ojos que se dejaban engañar por las ilusiones. Esta representación fija de la alteridad resultó ser el hilo con el que se deshacía todo el tejido de ideas y opiniones que forman una cultura.

Los pioneros de lo que luego se llamaron teorías postcoloniales iniciaron su revisión crítica de muchas concepciones erróneas de la cultura occidental tirando precisamente de este hilo para desembrollar lo que se ocultaba bajo el estereotipo. Frantz Fanon analizó profundamente las pautas de comportamiento de sus congéneres de color en las Antillas y denunció las convicciones sociales que la cultura blanca imponía en las colonias. Especialmente reveladora es su descripción de la gran influencia que las producciones de la cultura colonizadora tenía en la educación de los niños de color: "A drama is enacted everyday in colonized countries (...) In children's magazines, comics and adventure stories (...) the white adventurer overcomes the Negroes and Indians (...)." (1986: 140) Los habitantes de las Antillas, a su vez, identifican al "Negro" con el africano, reproduciendo el mismo método de reducción a la condición salvaje que los medios culturales blancos. El color de la piel, por tanto, no es totalmente concluyente. La misma gente de color no se identifica con el "Salvaje" de las historias de aventuras, sino con el héroe blanco.

Con este ejemplo, Fanon pone de manifiesto que el proceso de formación de un estereotipo es más complejo que la simple separación racial. A veces personajes blancos (los piratas, por ejemplo) cobran una representación fija de cualidades humanas rechazadas. La explicación psicológica de C. G. Jung sobre este proceso, mencionada por Fanon, es que el hombre traspasa todas las características que considera negativas e instintivas hacia otros seres en los que se hacen esenciales, para lo cual cualquier diferencia sirve de pretexto.

Este ataque al estereotipo de naturaleza psicológica se ve completado por la descripción histórica de la creación de figuras de la alteridad que Edward Said aplica a la visión europea de Oriente. Peter Hulme, por su parte, se centra en la visión mediatizada de los indios americanos. Ambos dudan de la verdad histórica de crónicas y relatos de viajes, que por razones obvias tendían hacia la exageración propagandística de las nuevas tierras, que justifican el riesgo económico y físico que suponían. Al mismo tiempo, era difícil no caer en comparaciones de las nuevas experiencias con las preconcepciones de las audiencias europeas, como indica Stephen Greenblatt (1994: 23). Este autor puso de relieve la importancia de la tradición de la literatura de viajes, citando en concreto la obra medieval Sir John Mandeville's Travels o los viajes de Marco Polo. Greenblatt introduce un concepto fundamental en la explicación (y posterior desmitificación) del estereotipo: el concepto de "maravilla". En él se resumen las influencias de las expectativas de los europeos en sus descripciones de las tierras que visitaban, y que impregnaban muchas de las explicaciones de fenómenos que no entendían. Los relatos, destinados a un público a miles de kilómetros, pretendían maravillar al lector con comparaciones extremas y exageraciones respecto de su propia realidad. El uso de la mimesis acentúa la condición literaria (por contraposición a histórica, verdadera) de muchas de estas crónicas. Esta indefinición sirve a Eric Cheyfitz para exponer un especial énfasis en la contraposición entre historia y relato pseudoliterario, entre sentido literal del lenguaje y sentido figurado, es decir, metafórico (1991: xvi). El autor denuncia la distinción espacial entre ambos casos: el sentido literal se presupone propio del conocimiento "científico" occidental, mientras que el uso metafórico del lenguaje se atribuye a las culturas primitivas que no poseen ese método empírico. Esto caracterizó la expresión estereotípica de los colonizados con metáforas sobre el orden natural o sobre las máquinas que la tecnología occidental presentaba a sus ojos.

Odisea, $\mathrm{n}^{\mathrm{0}} 2,2002$ 
Es en este punto de contraposición donde Homi Bhabha y Alfred Arteaga retoman el tema. La antítesis, considerada como parte fundamental del método occidental de conocimiento, aparece como uno de los principales factores que formaron la imagen de la alteridad. La afirmación de la identidad propia de una sociedad o de un individuo necesita una figura antitética para fortalecerse y justificarse. La cultura occidental, para acentuar su imagen de modernidad y civilización califica cualquier pueblo extraño de "salvaje" y "bárbaro". La esencialización de la diferencia culmina si además existe algún rasgo distinto visible, como el color de la piel, afirma Bhabha (1994: 78-81). Entonces el estereotipo se convierte en un objeto palpable (más fácil de aprehender) y, como describe Arteaga, totalmente ajeno, que concentra todo aquello que se considera nefasto en la cultura propia. Esta diferencia esencial, sugiere Arteaga (1994: 15-20), se lleva a todos sus efectos: la separación es artística, científica, religiosa e histórica. El "Otro" representa lo feo, la superstición y una etapa superable del desarrollo hacia la civilización colonizadora.

Una vez creado, el estereotipo debe permanecer ajeno. Para fijarse y mantenerse debe repetirse constantemente, especifica Bhabha (1994: 77). La producción cultural no puede permitir una representación diferente, que podría minar la construcción. Junto con este método de desmitificación del estereotipo, Bhabha pone un énfasis importante en el "Third Space", "margin", "non-place", en definitiva, el espacio entre las representaciones antitéticas donde la metáfora y la metonimia desarbolan los sentidos estrictos y literales de las representaciones antitéticas. Arteaga, por su parte, considera la síntesis como medio para evitar la contraposición de la alteridad y propone una literatura dialéctica, híbrida, políglota y multicultural, una literatura de frontera; también, casualmente, un espacio éste más ideológico que real.

Pues bien, estas dos estrategias, metáfora y frontera dialéctica son las que Golding utiliza fundamentalmente para desarticular los estereotipos que tanto le molestaban. El grupo de sus primeras novelas en el que aquí nos vamos a centrar presenta aspectos en común relacionados con la desmitificación de estereotipos a pesar de que el resto de temas, situaciones y personajes de las novelas sean tan variados. Uno de estos aspectos es la presentación de momentos claves en la historia humana. Al observar estos periodos esenciales de evolución del hombre, somos testigos de los terribles enfrentamientos dialécticos, mucho más acentuados que en periodos de calma evolutiva. No sólo se describen posibles acontecimientos pasados como en The Spire y The Inheritors, sino que además se somete a análisis el comportamiento del hombre en el presente (Pincher Martin) e incluso en un futuro indeterminado (Lord of the Flies). Las novelas son puntuales: el momento de la acción es breve, con lo que el choque de ideologías se acelera y profundiza.

La elaboración poética y estilística de la prosa goldinguiana presta atención al segundo de los aspectos, el carácter metafórico del lenguaje. Ciertos objetos palpables del espacio de las novelas toman una significación fundamental pero nunca unívoca. Los significados son variables, ricos, destinados tal vez a una interpretación psicoanalítica o dirigidos directamente al subconsciente humano. La metáfora resulta una asociación de impresiones en estado puro, es decir, no mediatizada por las explicaciones de la razón humana. La concha y la cabeza de cerdo en Lord of the Flies, la cascada de The Inheritors, la estatua de roca en Pincher Martin o la misma catedral de The Spire son ejemplos de estos elementos metafóricos.

La primera novela, Lord of the Flies, termina con el mito tan manido de la reinvención de la civilización en tierras nuevas. Pero los Ralph y Jack de Golding se enfrentan con un conflicto mucho mayor que los estereotipados personajes de Ballantyne. Situada en una 
futura guerra, los niños de Lord of the Flies se encuentran en el momento crítico en que, salidos de una situación de conflicto armado, pueden emplear sus convicciones culturales para crear una nueva civilización, libre de los defectos de sus mayores. En este sentido, la situación es similar a la de multitud de relatos anteriores. Pero los chicos se ven incapaces de comportarse "civilizadamente". A pesar de su educación británica, retroceden hasta un estado social equiparable al de los reductores de cabezas de las historias que seguramente habían leído. Las reuniones en torno a la concha se suceden, se discuten los temas que más les afectan: el enfrentamiento ideológico es encarnizado. Piggy se pregunta, apelando a la razón: "Which is better - to be a pack of painted niggers like you are, or to be sensible, like Ralph is?" (199). Pero la contestación no se hace esperar: los niños obedecen ciegamente a su nuevo jefe Jack y realizan bailes rituales y sacrificios en un proceso que muchos críticos han calificado como regresión ${ }^{1}$. En la confrontación entre quienes se comportan como salvajes y quienes defienden la ideología democrática heredada, los "demócratas" pretenden, como sus mayores, hacer esencial la diferencia, como ante cualquier estereotipo. Ralph protesta: “Well, we won't be painted', said Ralph, 'because we aren't savages"” (191). El cambio de apariencia representa para Ralph y Piggy un cambio en esencia. Los otros chicos dejan de ser británicos, civilizados e individuos; sólo son "anonymous savages" $(155)^{2}$.

Sin embargo, pese al uso extremo de las condiciones que forman el estereotipo, Ralph y Piggy no convencen y el resto de los niños se unen a la tribu de Jack. El estereotipo del salvaje salta hecho pedazos cuando, al final de la novela, el oficial británico desembarca en la isla y observa un grupo de niños ingleses pintados como salvajes. La violencia, la obediencia brutal, los sacrificios supersticiosos, no son privativos de "otros" pueblos, sino algo inherente al hombre. Pero el enfrentamiento dialéctico no permite conclusión alguna. La duda sobre quién es más salvaje, las tribus africanas o el oficial con su barco de guerra permanece sin resolver incluso una vez terminada la novela.

Bajo este nivel dialéctico la narrativa posee toda una serie de símbolos y metáforas que nos recuerdan que existe una realidad más profunda en el comportamiento humano. El principal causante de que los niños se comporten como una horda es el miedo irracional a una "bestia" cuya existencia no es segura. La bestia, la cabeza de cerdo y la tormenta, frente a la concha democrática y al fuego simbólico de la civilización (cuya extinción permiten los niños), profundizan en el subconsciente humano y sus realidades irracionales, evocando un mundo que escapa a cualquier representación. La metáfora de la bestia (curiosamente, un cadáver humano) no sólo aparece del lado de unos salvajes estereotipados: todos los hombres son iguales ante el pánico irracional. El símbolo del sacrificio al miedo, la cabeza de cerdo, que podía aplicarse a los salvajes, produce el mismo terror que una metáfora de la tecnología, como es el piloto muerto que desciende para convertirse en la bestia. Todo ello ante la mirada impasible del mar y de la naturaleza de la isla, como para generalizar y universalizar estos comportamientos a todo el género humano, a su propia naturaleza.

Los elementos naturales vuelven a ser testigos mudos del proceso dialéctico entre dos etapas de la evolución humana en The Inheritors, que se desarrolla en la época del hombre

\footnotetext{
${ }^{1}$ Oldsey y Weintraub (1965) llaman a Lord of the Flies "a social allegory of human regresion", mientras que Kinkead-Weekes y Gregor (1984) usan el término "reversion”. Véase también Virginia Tiger (1974: 42), Johnston (1980), Boyd (1988), Whitley (1970), y Paulette Michel-Michot en Delbaere (1991).

${ }^{2}$ Véase Hulme (1986) y Mary Louise Pratt (1993) sobre la homogeneización a que se somete a los diversos pueblos colonizados.
}

Odisea, $n^{\circ}$ 2, 2002 
de Neandertal y de la llegada de la nueva especie Homo sapiens ${ }^{3}$. En este pasado prehistórico, la catarata, como metáfora del irresistible progreso humano, es el centro del espacio donde se desenvuelve la acción. De nuevo se repiten las condiciones de Lord of the Flies: un espacio aislado, campo de batalla entre concepciones de la realidad, todo ello para desmontar la imagen estereotípica que circulaba de los hombres de Neandertal como "monstruos". Golding hace que el monstruo tome la palabra: la narración se hace desde el punto de vista de Lok, uno de los neandertales.

El cambio de punto de vista implica una preocupación por la representación de la alteridad. Siguiendo las pautas del estereotipo, Golding atribuye a Lok una expresión metafórica, intransitiva, pero tamizada siempre por la medida humana. Su pensamiento lógico es deficiente, pero su inocencia se demuestra en la capacidad para emitir juicios morales. Describe a los Homo sapiens por oposición; al seguir el rastro oloroso de uno de ellos, afirma: "With the scent of the other I am other. I creep like a cat. I am frightened and greedy. I am strong" (97). Lok demuestra por tanto unas facultades más avanzadas que las de nuestros antepasados más directos para comprender el punto de vista de otros seres. En esta imposibilidad de identificarse mínimamente con el "Otro" se encuentra la formación del estereotipo. Los Homo sapiens, atenazados por el miedo, ni siquiera se molestan en acercarse pacíficamente a Lok y los suyos. Simplemente destruyen aquello que no conocen. En efecto, resulta tristemente cierta la afirmación de Wells en su historia "The grisly folk": "We cannot conceive in our different minds the strange ideas that chased one another through those queerly shaped brains." ${ }^{5}$ A nuestros antepasados, el pánico les lleva a levantar toda una serie de barreras de separación contra quienes pronto denominan "demonios". Nuestros ancestros les atribuyen cualidades mágicas y realizan toda suerte de ritos para ahuyentarlos. En el último capítulo, uno de los Homo sapiens, Tuami, atribuye cualidades mágicas y tabúes a estos seres que han pasado a encarnar esencialmente el mal, aunque en la novela los únicos culpables de la serie de atrocidades narradas son los mismos Homo sapiens. Sin embargo, su pensamiento (conocimiento) racional es capaz de justificar todas sus actuaciones: "What else could we have done?" (227), se pregunta retóricamente Tuami.

Al final, los Homo sapiens consiguen superar el escollo a su progreso que presentaba la catarata, una caída de agua definida como "upward waterfall", donde Golding pone en juego metafóricamente todo el conflicto de la novela. Lo que desde la justificación lógica de nuestros ancestros supone un avance hacia una nueva era (contra corriente), ha tenido en realidad unos orígenes tenebrosos, llenos de crímenes atroces, incluido el canibalismo. Las armas que se consideraban prueba de mayor nivel de civilización, un conocimiento lógico más desarrollado y mejor tecnología, implican a la vez un uso menos ético. Podríamos pensar que The Inheritors no es más que otra historia del mito del "buen salvaje", apegado a la naturaleza e inocente por definición, pero al final de la novela Lok es capaz de planear una estrategia para liberar a una de las neandertales: las maquinaciones desmienten su estado de inocencia esencial. La conclusión no nos salva de disquisiciones dialécticas. Golding pone hábilmente los dos puntos de vista a nuestro alcance. Una visión unívoca parece incorrecta, por lo que el lector tiene muchas dudas a la hora de identificarse con el

\footnotetext{
3 El choque entre dos civilizaciones y sus consecuencias ha hecho que autores como Kevin McCarron (1994: 10) hayan observado el tema del colonialismo en esta novela. También sugirieron temas parecidos Johnston (1980: 32) y Boyd (1988: 42).

${ }^{4}$ Golding mismo hace referencia a esta idea en su prólogo a la novela donde cita un fragmento extraído de The Outline of History, de H.G. Wells.

${ }^{5}$ Wells, H.G. Selected Short Stories. Harmondsworth: Penguin, 1982, p. 289.
} 
lenguaje lógico de Tuami o el cariñoso y atrayente lenguaje metafórico, lleno de frescura, de Lok: ambas son expresiones humanas. Este estado de inocencia contrarresta la convicción de Tuami, quien se muestra confiado en el avance humano, afirmando, en una expresión casi romántica del individualismo y posibilidad de perfección humana: "A fair wind, steerage way and plenty of water all around-what more could a man want?" (224).

No obstante, en caso de que alguien hubiera extraído una conclusión simple y directa de su novela, Golding parece retomar el tema del navegante individualista, solitario y satisfecho al comienzo de la siguiente, Pincher Martin. Christopher Hadley Martin se encuentra solo frente al mar, aunque en peores circunstancias que Tuami, y va a experimentar la máxima expresión del individualismo humano: terminará solo en una isla donde deberá construir su propia civilización à la Robinson Crusoe. Nuevamente una situación extrema y conflictiva ocupa el centro de la narración, donde se pone a prueba la afirmación de la perfección de las capacidades racionales del hombre. Como representante de la individualidad más acusada, Christopher Martin sobrevalora la capacidad de su inteligencia y su razón como herramientas de supervivencia, y afirma: "But I've got health and education and inteligence. I'll beat you" (77). Pronto tal individualismo presenta sus puntos débiles. Martin siente la necesidad de crear otro ser dentro de su isla. Construye un hombre con piedras, con la finalidad de poseer un sirviente, como lo fue Friday para Robinson. Martin demuestra que la afirmación de su identidad y situación no es posible sin la presencia de otros en quienes verse reflejado y de quienes diferenciarse. La utilización de este "Otro" como sirviente nos recuerda su percepción de los demás como objetos que mencionábamos en páginas anteriores. Sin embargo, el sirviente de Christopher parece más bien un enano deforme -"Dwarf"-, como Caliban en The Tempest. El náufrago utiliza un trozo de papel de aluminio para dar una cara al enano de metro y medio, pero progresivamente empieza a ver reflejada en él la presencia de su propio "yo", como se nos describe en la narración: "He went close to the dwarf and looked down at the head to see if he could find his face reflected there" (107). Esta figura de la alteridad creada por Martin mismo con objetos perceptibles pierde su valor desde este momento en que caen las barreras que los separan. El enano se relaciona directamente con el símbolo goldinguiano de lo oculto por la razón: la bodega oscura, donde Christopher esconde los temores irracionales de la infancia, encarnados en la figura de una vieja ${ }^{6}$. Martin lo expresa así: "She is the Dwarf. I gave her a silver head" (176). El enano, "Otro", se carga simbólicamente. Al mismo tiempo que niega la existencia de la parte deforme del ser humano, sucumbe ante la necesidad de contrapunto necesario sin el que el estado de civilización y racionalidad no puede definirse. Al contrario que Robinson, quien puede exteriorizar el mal en los salvajes del continente, Christopher fracasa en su intento de no llevarse a la isla todo lo negativo que su civilización le había dado. Aunque está convencido de su bondad natural y de su superior capacidad de raciocinio, debe enfrentarse a la realidad de que esa misma inteligencia le ha hecho cometer atropellos: son los "flashbacks" que presencia durante la narración. El individualismo presenta su lado negativo: la explotación de los otros, a lo que Golding atribuye nuevas metáforas: la caja china, la boca, los charcos oscuros. La isla que para Robinson es un paraíso, para Christopher es un purgatorio ${ }^{7}$, donde la racionalidad y la voluntad sucumben ante el miedo y la explotación, y donde aparece una realidad muy influyente en el ser humano, el subconsciente irracional, cuya representación en el "Otro" ha perdido su validez.

\footnotetext{
${ }^{6}$ En la interpretación junguiana la imagen arquetípica de la mujer representa la parte femenina de la mente del hombre, el anima, que se identifica con el subconsciente (Jung, 1976).

7 Véase Virginia Tiger (1974: 128).
}

Odisea, $n^{\circ} 2,2002$ 
Esta utilización interesada de los demás se estudia más profundamente en The Spire (aunque también en menor medida en la novela que media entre ellas, Free Fall, que se articula en torno a las reflexiones de un hombre encerrado en una celda oscura). La culminación del proceso de estereotipación se muestra en la explotación del "Otro", como habían demostrado los diversos procesos de colonización y conquista, y como preconizaba el sistema clasista europeo. Tal vez por esto el proceso de desmitificación del estereotipo debe concluir con la denuncia de la explotación y el sacrificio de este "Otro". El deán Jocelin debe enfrentarse con sus actos inmorales durante la construcción de su visión, la torre y la aguja. Pero la narración no acaba ahí, sino que prosigue hasta que Jocelin reconoce su culpa y la realidad de esta parte oculta que él identifica con los demonios. El deán maneja como marionetas a quienes le rodean, para hacer "la voluntad de Dios", es decir, para construir la torre. Su propia voluntad se mezcla con la divina.

La novela retorna al conflicto de la valoración de lo que se considera la expresión más elevada de la civilización y tecnología medievales y denuncia la objetivación que sufren las personas a las que la construcción de la aguja destruye. De todos ellos, quien concentra todas las características del chivo expiatorio es Pangall. Debido a su deformidad se convierte en el centro de las burlas de los obreros, que las utilizan como vía de escape a su terror. Éstos son retratados como una turba salvaje y sin escrúpulos. "Why me? Isn't there anyone else? Why must they make a fool of me?" (19), se pregunta Pangall, que acaba asesinado y enterrado en los cimientos. Su reducción a un objeto es tan efectiva en la mente de Jocelin que de él sólo le queda un leve recuerdo del muérdago simbólico del sacrificio. Jocelin observa: “ (...) there was a twig lying across his shoe, with a rotting berry that clung obscenely to the leather (...) The berry and the twig could not be forgotten (...)" (95). Igual que en Pincher Martin, la figura del "Otro", Pangall, está asociada con el elemento simbólico que se refiere al lado oscuro de la sociedad: si Christopher Martin relaciona al enano con la anciana de la bodega oscura, Pangall es enterrado justo en el pozo excavado en el transepto, que concentra todas las connotaciones simbólicas de oscuridad y profundidad.

La torre se convierte en la gran metáfora de las débiles bases sobre las que muchas veces se asientan los triunfos más laureados de la civilización. Jocelin se pregunta: "What holds it up, Roger? I? (...) Or is it poor Pangall, crouched beneath the crossways, with a sliver of mistletoe between his ribs?" (212). Jocelin da el paso que ni Christopher ni Sammy pudieron superar, al aceptar que en realidad el hombre posee una parte fuera del control racional que intenta exteriorizar en los demás. La aguja conecta lo más elevado de comportamiento humano (su tendencia hacia la divinidad) con toda la miseria que se esconde en los cimientos. El deán se da cuenta por fin de que es algo más que una voluntad o una visión supuestamente divina: "I'm a building with a vast cellerage where the rats live (...)," (210) y deduce que es una condición de todo ser humano: "What's a man's mind, Roger? Is it the whole building, cellerage and all? (213). La aceptación de que la catedral (identificada con el hombre desde el principio de la novela) simboliza una conexión entre el cielo (lo elevado de la condición humana) y el subsuelo (la parte más oscura y oculta de la naturaleza humana) establece, en definitiva, una relación entre la alteridad y la parte oscura del hombre. Se elimina así la posibilidad de separación entre unos seres puramente perversos, en quienes se proyecta todo el mal y la misma sociedad que crea la ilusión de la civilización negando una parte esencial del hombre que existe nos guste o no.

Frente a la construcción de la cultura occidental basada en una visión parcial de la realidad pero justificada como poseedora de la verdad, las situaciones dialécticas presentan 
diversos significados e interpretaciones a nivel racional (argumentación), mientras que la fluidez significativa de las metáforas se acerca mucho más a toda esa parte de nuestro ser no dominada por la razón. De esta manera, la imagen del hombre que nos presenta Golding es mucho más global y completa que la triste separación entre "civilizados" y "salvajes", al mismo tiempo que saca a la superficie las debilidades de la construcción estereotípica.

El autor comienza con una crítica a la exteriorización del mal que establece la sociedad inglesa y la civilización occidental en general y acaba adquiriendo tintes de un ataque a la supuesta superioridad de las sociedades civilizadas. Éstas tradicionalmente han justificado su apropiación de territorios ajenos por la necesidad de "civilizar" a esos otros pueblos que permanecían en estado "salvaje" y no habían "visto la luz". Para ello utilizan la cultura y las representaciones estereotípicas de estas gentes de tal forma que los pueblos primitivos realizan una función doble: por un lado concentran todo lo que se considera negativo en las sociedades "civilizadas" y por otro, define la misma "civilización" por contraste.

Golding arremete primero contra los estereotipos de los "primitivos" y la exteriorización del mal, minando las barreras que encapsulan las representaciones estereotípicas de los salvajes de Lord of the Flies o de los monstruos de The Inheritors. La "bestia" de Lord of the Flies y el "enano" de Pincher Martin, son para Golding figuras tan erróneas como la representación de los salvajes o primitivos que aparecen en Lord of the Flies o The Inheritors. Establecer una unión entre aquello que antes era alteridad absoluta y aquello que deja a un lado la cultura dominante significa destrozar toda la construcción que ha hecho que se proyecte ese "lado oscuro" en los estereotipos de los salvajes.

Pero a Golding no le basta con reconocer el error en que caen las representaciones estereotípicas del "Otro" cuando se proyecta en ellas todo lo negativo de una cultura. También tiene que mostrar cómo funciona la creación de un estereotipo. Por eso se sitúa dentro de la figura de la alteridad. En The Inheritors, vemos a nuestros antepasados en el momento en que crean esta imagen de monstruos que se basa principalmente en la imaginación, ya que los "new men" (los Homo sapiens) no conocen realmente a los neandertales. Estos hombres nuevos se sirven del lenguaje y de la razón para ocultar sus miedos debajo de una línea simbólica de oscuridad y bajo la denominación de demonios "devils"-. Los neandertales quedan como figuras de la alteridad en las que se proyectan todos los temores irracionales de la nueva sociedad, creando así un estereotipo (el de los ogros) que perdura hasta nuestros días.

En su historia The Scorpion God, Golding ejemplifica cómo la visión dominante puede estar equivocada y a pesar de eso, imponerse sobre la concepción del "Otro", simplemente porque éste es menos poderoso como en The Inheritors, o menos numeroso como en esta novela corta. Posteriormente, el cambio de concepción de la realidad puede acarrear el cambio completo de cultura. En definitiva, el autor demuestra la relatividad de las presunciones de superioridad de unas civilizaciones sobre las otras. Al adoptar la supuesta perspectiva del "Otro", Golding está reafirmando su creencia en la validez de las diferentes formas de conceptualizar el mundo que tienen las diversas culturas y los diferentes individuos, es decir, está atacando una explicación unívoca y reduccionista de la realidad. Esto significa en literatura el desplazamiento del narrador omnisciente por unos narradores que observan la realidad desde una perspectiva propia y subjetiva, tanto que puede incluso tratarse de alguien cuya percepción de la realidad esté condicionada por sus características mentales (Lok en The Inheritors), sus intereses particulares (Christopher en Pincher Martin) o sus obsesiones (Jocelin en The Spire). Con esto elimina el autor la presunta fiabilidad de una representación no subjetiva de la realidad. Esta desmitificación de 
estereotipos y la relativización de las concepciones sobre el mundo sitúan a Golding como antecesor de las ideas que han impulsado las teorías postcoloniales.

\section{BIBLIOGRAFÍA}

Arteaga, A., ed. 1994. Nation and Ethnicity in the Linguistic Borderlands. Durham and London: Duke Univerisity Press.

Ballantyne, R. M. 1990. The Coral Island. Oxford \& New York: O.U.P.

Bhaba, H. K. 1994. The Location of Culture. London \& New York: Routledge

Boyd, S. 1988. The Novels of William Golding. Brighton: Harvester.

Cheyfitz, E. 1991. The Poetics of Imperialism. New York \& Oxford: O.U.P.

Defoe, D. 1993. Robinson Crusoe. Ware: Wordsworth.

Fanon, F. 1986. Black Skin, White Masks. London \& Sydney: Pluto Press.

Golding, W. 1958. Lord of the Flies. London: Faber \& Faber.

. 1961. The Inheritors. London: Faber \& Faber.

. 1956. Pincher Martin. London: Faber \& Faber

. 1961. Free Fall. London: Faber \& Faber

. 1964. The Spire. London: Faber \& Faber

. 1973. The Scorpion God. London: Faber \& Faber,

. 1967. The Hot Gates and Other Occasional Pieces. New York: Pocket books.

. 1982. A Moving Target. London: Faber \& Faber.

Greenblatt, S. 1994. Marvelous Possessions. The Wonder of the New World. London \& New York: Routledge.

Hulme, P. 1992. Colonial Encounters. London \& New York: Routledge.

Johnston, A. 1980. Of Earth and Darkness: The Novels of William Golding. Columbia: University of Missouri Press.

Jung, C.G. 1997. Arquetipos e Inconsciente Colectivo. Barcelona: Piados. 1976. El Hombre y sus Símbolos. Barcelona: Caralt.

Kinkead-Weekes, M. and I. Gregor. 1984. William Golding, A Critical Study. London: Faber \& Faber.

McCarron, K. 1994. William Golding. Plymouth: Northcote House Publishers Ltd.

Michel-Michot, P. 1991. "The myth of innocence: Robinson Crusoe, The Coral Island and Lord of the Flies". William Golding. The Sound of Silence. A Belgian Tribute on his Eightieth Birthday. Ed. J. Delbaere. Liège: Liège Language and Literature.

Oledsey, B. S. and S. Weintrub. 1965. The Art of William Golding. New York: Harcourt, Brace \& World. 
Pratt, M. L. 1993. Imperial Eyes. Travel Writing and Transculturation. London \& New York: Routledge.

Said, E. 1979. Orientalism. New York: Vintage Books.

1994. Culture and Imperialism. New York: Vintage Books.

Shakespeare, W. 1963. La Tempestad. Madrid: Editora Nacional.

Tiger, V. 1974. William Golding: The Dark Fields of Discovery. London: Calder \& Boyars.

Wells, H.G. 1972. The Outline of History. London: Cassell . 1981. Selected Short Stories. Harmondsworth: Penguin.

Whitley, J. S. 1970. Golding: Lord of the Flies. London: Edward Arnold. 